\title{
A Validation Study for the Utility of Serum microRNA as a Diagnostic and Prognostic Marker in High-Grade Osteosarcoma Patients
}

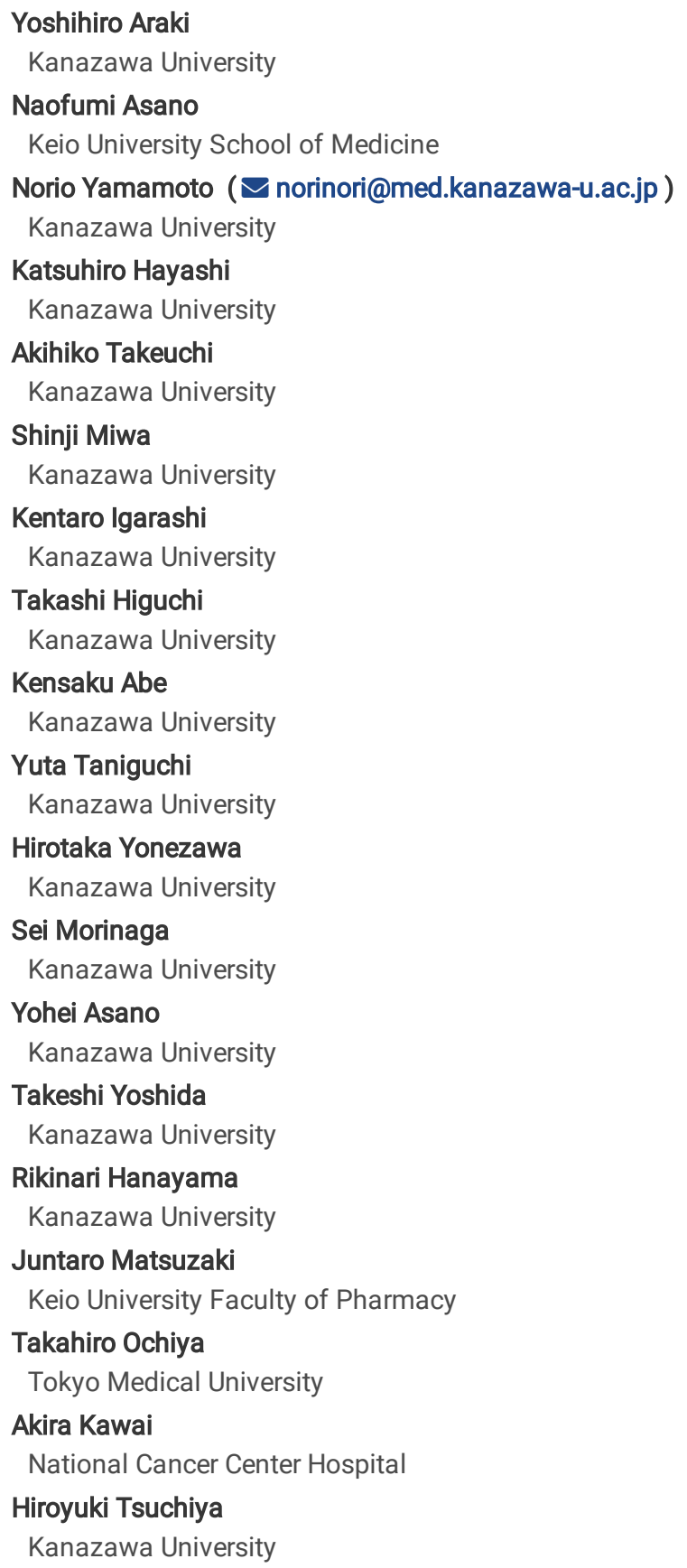

\section{Research Article}

Keywords: Validation, Utility, Serum miRNA, Osteosarcoma, Diagnosis, Prognosis, Marker

Posted Date: February 23rd, 2022

DOI: https://doi.org/10.21203/rs.3.rs-1371025/v1

License: (c) (i) This work is licensed under a Creative Commons Attribution 4.0 International License. Read Full License 


\section{Abstract}

In our previous study, high-grade osteosarcoma advanced locally and promoted metastasis by suppressing osteoclastogenesis via the upregulation of miR-146a-5p. Another 14 miRNAs in small extracellular vesicles were also detected 5 or more times as frequently in high-grade malignancy as in low-grade malignancy. However, the utility of these 15 miRNAs for determining the prognosis or diagnosis of osteosarcoma has not been validated in the clinical setting. We therefore aimed to assess the utility of these serum miRNAs as prognostic and diagnostic markers. Thirty osteosarcoma patients were retrospectively reviewed, excluding those with metastasis at presentation. The survival was compared according to the serum miRNA values. In addition, to confirm diagnostic competency for osteosarcoma, the serum miRNA values were compared with those in other bone tumor patients $(n=112)$ and healthy controls $(n=275)$. Osteosarcoma patients with high serum miRNA values paradoxically showed a better survival than those with low values. These patients also had higher values of serum miR-1261 than those with benign or intermediate-grade bone tumor. Serum miR-146a-5p may not be useful marker for determining the prognosis, and a larger investigation will be required to clarify its actual utility. In contrast, serum miR-1261 may be useful for differentiating benign or intermediate-grade bone tumor.

\section{Introduction}

MicroRNA (miRNA) consists of 20 to 30 bases and plays an important role in fostering communication among cells in various creatures ${ }^{1}$. Tumor cells also affect the surrounding normal cells via miRNAs, encouraging changes in the tumor microenvironment to facilitate progression ${ }^{2-6}$. These miRNAs have been reported to be transmitted to the target cells via tumor-derived small extracellular vesicles ${ }^{3-6}$. Thus, these miRNAs carried by tumor-derived small extracellular vesicles in circulating blood may be useful as prognostic or diagnostic markers of tumors ${ }^{7-11}$.

Sarcoma is a rare cancer, and osteosarcoma usually occur in children, adolescents and young adults ${ }^{12,13}$. The diagnosis of osteosarcoma generally depends on the histological findings of a biopsy specimen. The prognosis of these patients has been reported to be associated with the presence of metastasis at presentation, delayed initiation of treatment, a high level of alkaline phosphatase, combination of pretreatment neutrophil count and platelet-lymphocyte ratio, poor response to chemotherapy, and mature osteoclast number in a biopsy specimen ${ }^{14-19}$ Recently, several serum miRNAs associated with the prognosis or diagnosis of osteosarcoma have been reported ${ }^{20-25}$. Circulating miR-663a, miR25-3p, miR-487a, miR-493-5p, miR-501-3p, and miR-502-5p have been reported to be useful as diagnostic and prognostic biomarkers for osteosarcoma, and blood tests are easy and simple to perform for patients.

In our previous study, high-grade osteosarcoma advanced locally and promoted metastasis through the suppression of osteoclastogenesis via the upregulation of miR-146a-5p. We also identified several other miRNAs (miR-1260a, miR-487b-3p, miR-6720-3p, miR-1260b, miR-4758-3p, miR-46903p, miR-4286, miR-6765-3p, miR-1261, miR-7975, miR-4664-5p, miR-3907, miR-7977, miR-1273c) that were included in small extracellular vesicles derived from high-grade osteosarcoma cells at rates five times or greater than in low-grade osteosarcoma cells ${ }^{26}$. However, the utility of these 15 miRNAs for determining the prognosis or diagnosis of high-grade osteosarcoma has not yet been validated in the clinical setting.

We therefore investigated the association of these miRNAs with the prognosis of osteosarcoma patients and their potential utility in the diagnosis of osteosarcoma and differentiation from other bone tumors.

\section{Results}

\section{Patient characteristics}

The study population included 20 male and 10 female patients with a mean age of 24 (range, 10-68) years old. The histological findings were high-grade osteosarcoma in all cases. Tumors were located at the trunk in 3 patients and appendicular skeleton in 27 patients. No distant metastasis was observed at presentation in any cases.

The mean tumor size was $9.7 \mathrm{~cm}$ in the greatest dimension (range, 6-17.5 cm). The tumor stage was IIA in 13 patients, IIB in 14 patients, and III in 3 patients according to the American Joint Committee on Cancer (AJCC) 8th staging classification. All of the patients underwent surgical treatment. Chemotherapy was performed in 29 patients, and 2 patients underwent radiotherapy. The mean follow-up period was 55 (range, 17-81) months. Local recurrence was observed in 1 patient at 48 months after surgery. Distant metastasis was observed in 11 patients at a mean follow-up period of 23 (range, 10-37) months. Four patients died of their disease at a mean follow-up period of 39 (range, 26-47) months. At the final follow-up, a total of 21 patients were free from disease.

The characteristics of the patients with other types of bone tumors were as follows: 8 male and 7 female patients with a mean age of 27 (range, 6 48) years old with osteochondroma, 7 male and 4 female patients with a mean age of 42 (range, 11-75) years old with enchondroma, 7 male and 3 female patients with a mean age of 31 (range, 12-68) years old with fibrous dysplasia, 15 male and 13 female patients with a mean age of 35 (range, 10-67) years old with giant cell tumor of bone, 3 male and 2 female patients with a mean age of 52 (range, 36-63) years old with synovial chondromatosis, 1 male and 2 female patients with a mean age of 16 (range, 15-18) years old in osteoblastoma, 19 males and 7 female patients with a mean age of 27 (range, 8-65) years old with Ewing sarcoma, 6 male and 2 female patients with a mean age of 60 (range, 26-92) years old 
in chordoma, 3 male and 3 female patients with a mean age of 48 (range, 19-76) years old in chondrosaroma (Grade2-3). The healthy controls were 150 male and 125 female individuals with a mean age of 52 (range, 35-80) years old.

\section{The survivals}

The optimal cut-off points and the areas under the curve (AUC) of the miRNAs (miR-146a-5p, miR-1260a, miR-487b-3p, miR-6720-3p, miR-1260b, miR-4758-3p, miR-4690-3p, miR-4286, miR-6765-3p, miR-1261, miR-7975, miR-4664-5p, miR-3907, miR-7977, and miR-1273c), predicting death from disease, distant metastasis, or freedom from disease, are shown in Table 1.

Table 1

The optimal cut-off values, AUC, sensitivity, and specificity of variables to predict death from disease, distant metastasis, or freedom from disease.

\begin{tabular}{|c|c|c|c|c|c|c|c|c|c|c|c|c|}
\hline & \multicolumn{4}{|c|}{ Overall survival } & \multicolumn{4}{|c|}{ Distant metastasis-free survival } & \multicolumn{4}{|c|}{ Disease-free survival } \\
\hline & $\begin{array}{l}\text { Cut- } \\
\text { off } \\
\text { value }\end{array}$ & AUC & Sensitivity & Specificity & $\begin{array}{l}\text { Cut- } \\
\text { off } \\
\text { value }\end{array}$ & AUC & Sensitivity & Specificity & $\begin{array}{l}\text { Cut- } \\
\text { off } \\
\text { value }\end{array}$ & AUC & Sensitivity & Specificity \\
\hline $\begin{array}{l}\text { miR- } \\
146 a- \\
5 p\end{array}$ & 5.743 & 0.904 & $100 \%$ & $75 \%$ & 6.693 & 0.67 & $57.90 \%$ & $72.70 \%$ & 5.743 & 0.614 & $100 \%$ & $33.30 \%$ \\
\hline $\begin{array}{l}\text { miR- } \\
1260 a\end{array}$ & 5.743 & 0.865 & $96.20 \%$ & $75 \%$ & 6.693 & 0.775 & $57.90 \%$ & $90.90 \%$ & 6.019 & 0.757 & $91 \%$ & $55.60 \%$ \\
\hline $\begin{array}{l}\text { miR- } \\
487 b- \\
3 p\end{array}$ & 5.743 & 0.894 & $100 \%$ & $75 \%$ & 6.693 & 0.742 & $57.90 \%$ & $90.90 \%$ & 6.257 & 0.672 & $81 \%$ & $55.60 \%$ \\
\hline $\begin{array}{l}\text { miR- } \\
6720- \\
3 p\end{array}$ & 5.743 & 0.875 & $92.30 \%$ & $75 \%$ & 5.743 & 0.656 & $94.70 \%$ & $36.40 \%$ & 5.743 & 0.741 & $100 \%$ & $55.60 \%$ \\
\hline $\begin{array}{l}\text { miR- } \\
1260 \mathrm{~b}\end{array}$ & 5.743 & 0.846 & $96.20 \%$ & $75 \%$ & 6.693 & 0.708 & $78.90 \%$ & $63.60 \%$ & 6.421 & 0.725 & $90.50 \%$ & $55.60 \%$ \\
\hline $\begin{array}{l}\text { miR- } \\
4758- \\
3 p\end{array}$ & 5.743 & 0.875 & $96.20 \%$ & $75 \%$ & 6.693 & 0.742 & $57.90 \%$ & $90.90 \%$ & 6.09 & 0.783 & $85.70 \%$ & $66.70 \%$ \\
\hline $\begin{array}{l}\text { miR- } \\
4690- \\
3 p\end{array}$ & 7.346 & 0.43 & $52 \%$ & $75 \%$ & 7.333 & 0.505 & $52.60 \%$ & $80 \%$ & 6.019 & 0.589 & $95 \%$ & $33.30 \%$ \\
\hline $\begin{array}{l}\text { miR- } \\
4286\end{array}$ & 7.349 & 0.538 & $46.20 \%$ & $100 \%$ & 7.378 & 0.689 & $52.60 \%$ & $90.90 \%$ & 7.349 & 0.709 & $52.40 \%$ & $88.90 \%$ \\
\hline $\begin{array}{l}\text { miR- } \\
6765- \\
3 p\end{array}$ & 5.618 & 0.663 & $100 \%$ & $50 \%$ & 6.693 & 0.703 & $57.90 \%$ & $81.80 \%$ & 6.019 & 0.593 & $90.50 \%$ & $44.40 \%$ \\
\hline $\begin{array}{l}\text { miR- } \\
1261\end{array}$ & 9.231 & 0.702 & $76.90 \%$ & $75 \%$ & 9.231 & 0.589 & $78.90 \%$ & $45.50 \%$ & 9.231 & 0.63 & $81.00 \%$ & $55.60 \%$ \\
\hline $\begin{array}{l}\text { miR- } \\
7975\end{array}$ & 6.621 & 0.76 & $53.80 \%$ & $100 \%$ & 6.693 & 0.727 & $57.90 \%$ & $90.90 \%$ & 5.743 & 0.762 & $100 \%$ & $44.40 \%$ \\
\hline $\begin{array}{l}\text { miR- } \\
4664- \\
5 p\end{array}$ & 5.99 & 0.837 & $84.60 \%$ & $75 \%$ & 6.621 & 0.646 & $57.90 \%$ & $72.70 \%$ & 6.019 & 0.735 & $85.70 \%$ & $66.70 \%$ \\
\hline $\begin{array}{l}\text { miR- } \\
3907\end{array}$ & 5.743 & 0.846 & $92.30 \%$ & $75 \%$ & 6.693 & 0.732 & $52.60 \%$ & $90.90 \%$ & 5.743 & 0.783 & $100 \%$ & $55.60 \%$ \\
\hline $\begin{array}{l}\text { miR- } \\
7977\end{array}$ & 5.878 & 0.837 & $84.60 \%$ & $75 \%$ & 6.693 & 0.756 & $57.90 \%$ & $90.90 \%$ & 6.019 & 0.794 & $90.50 \%$ & $66.70 \%$ \\
\hline $\begin{array}{l}\text { miR- } \\
1273 c\end{array}$ & 7.645 & 0.567 & $30.80 \%$ & $100 \%$ & 7.271 & 0.416 & $47.40 \%$ & $63.60 \%$ & 7.394 & 0.529 & $61.90 \%$ & $55.60 \%$ \\
\hline
\end{tabular}

A comparison of the overall survival according to miRNA values was shown in Fig. 1. The patients with high serum values of miR-146a-5p, miR1260a, miR-487b-3p, miR-6720-3p, miR-1260b, miR-4758-3p, miR-6765-3p, miR-4664-5p, miR-3907, and miR-7977 were significantly associated with a better overall survival than those with low values (miR-1260a, $p<0.01$; miR-487b-3p, $p<0.01$; miR-6720-3p, $p<0.01$; miR-146a-5p, $p<0.01$; miR1260b, $p<0.01$; miR-4758-3p, $p<0.01$; miR-6765-3p, $p=0.01$; miR-4664-5p, $p=0.01$; miR-3907, $p<0.01$; miR-7977, $p=0.01$ ). In contrast, the patients with high serum values of miR-4690-3p, miR-4286, miR-1261, miR-7975, and miR-1273c were not associated with a better overall survival than those with low values. 
A comparison of the distant metastasis-free survival according to miRNA values was shown in Fig. 2. The patients with high serum values of miR1260a, miR-487b-3p, miR-6720-3p, miR-1260b, miR-4758-3p, miR-4286, miR-6765-3p, miR-7975, miR-3907, and miR-7977 were significantly associated with a better distant metastasis-free survival than those with low values (miR-1260a, $p<0.01 ;$ miR-487b-3p, $p<0.01 ;$ miR-6720-3p, $p<$ 0.01; miR-1260b, $p<0.01$; miR-4758-3p, $p<0.01$; miR-4286, $p=0.03$; miR-6765-3p, $p<0.01$; miR-7975, $p<0.01 ;$ miR-3907, $p=0.02 ;$ miR-7977, $p<$ 0.01). In contrast, the patients with high serum values of miR-146a-5p, miR-4690-3p, miR-1261, miR-4664-5p, and miR-1273c were not associated with a better distant metastasis-free survival than those with low values.

A comparison of the disease-free survival according to miRNA values was shown in Fig. 3. The patients with high serum values of miR-146a-5p, miR-1260a, miR-6720-3p, miR-1260b, miR-4758-3p, miR-4690-3p, miR-6765-3p, miR-1261, miR-7975, miR-4664-5p, miR-3907, and miR-7977 were significantly associated with a better disease-free survival than those with low values (miR-1260a, $p=0.02 ; \mathrm{miR}-6720-3 p, p<0.01 ; \mathrm{miR}-146 a-5 p, p<$ 0.01; miR-1260b, $p<0.01$; miR-4758-3p, $p<0.01$; miR-4690-3p, $p=0.01$; miR-6765-3p, $p<0.01$; miR-1261, $p=0.01$; miR-7975, $p<0.01$; miR-4664-5p, $p<0.01$; miR-3907, $p<0.01$; miR-7977, $p<0.01$ ). In contrast, the patients with high serum values of miR-487b-3p, miR-4286, and miR-1273c were not associated with a better disease-free survival than those with low values.

\section{Differentiation from other types of bone tumors}

A comparison of serum miRNAs values in patients with bone tumors and healthy controls was shown in Fig. 4. The values of serum miR-1261 in osteosarcoma patients tends to be higher than those in healthy controls $(p<0.01)$ and patients with benign (osteochondroma, $p=0.01$; enchondroma, $p=0.08$; fibrous dysplasia, $p=0.09$ ) or intermediate-grade bone tumor (giant cell tumor of bone, $p=0.02$ ), while the values of serum miR-1273c in osteosarcoma patients tended to be lower than those in patients with benign (osteochondroma, $p=0.10 ;$ enchondroma, $p=0.12$ ) or intermediate-grade bone tumor (giant cell tumor of bone, $p=0.08$ ) but were similar to those in healthy controls. The serum values of both miR-1261 and miR-1273c were not significantly associated with those in patients with other high-grade bone tumors. In contrast, the serum values of miR146a-5p, miR-1260a, miR-487b-3p, miR-6720-3p, miR-1260b, miR-4758-3p, miR-4690-3p, miR-4286, miR-6765-3p, miR-7975, miR-4664-5p, miR-3907, and miR-7977 in osteosarcoma patients were not markedly different from those in patients with benign bone tumors, intermediate-grade bone tumors, or other high-grade bone tumors, although the values were significantly higher than those in healthy controls $(p<0.01)$.

\section{Discussion}

Various miRNAs have been reported to be associated with the diagnosis and prognosis of osteosarcoma patients, but very few miRNAs are clinically used as specific markers for the disease $20-25,27$. As in the present study, retrospective validation study and even prospective studies concerning the actual utility of targeted miRNAs have sometimes uncovered unexpected outcomes in clinical settings, although the reasons for these findings have not yet been discussed in detail.

In our previous in vivo and in vitro studies, high-grade osteosarcoma advanced locally and promoted metastasis through the suppression of osteoclastogenesis via the upregulation of miR-146a-5p. High-grade human osteosarcoma cells (143B) secreted more small extracellular vesicles than low-grade human osteosarcoma cells (HOS), and contained a large amount of various kinds of miRNAs, including miR-146a-5p. The miR146a-5p suppressed osteoclastogenesis in the tumor bone microenvironment, and osteosarcoma patients with a small amount of mature osteoclasts on histology of a biopsy specimen had worse prognosis and higher rates of lung metastasis than those with a large amount of mature osteoclasts ${ }^{26}$. Thus, a poor prognosis was expected in our patients with high values of miR-146a-5p, compared with those with low values.

The other 14 miRNAs in the present study were also identified in the 143B cells $\geq 5$ times more frequently than in the HOS cells in our previous study. Similarly, the miRNAs secreted from high-grade malignant tumors were expected to show high values in the blood, compared to those from low-grade malignant tumors. Therefore, high serum miRNA values were expected to reflect a poor prognosis.

However, the present study showed unexpectedly contrary outcomes concerning the prognosis according to the miRNA values. Patients with high serum values of some miRNAs had a better survival than those with low values, possibly due to the small number of patients, the different cut-off values of miRNAs used, or the decomposition of miRNAs in the serum and inconsistency of miRNA values in the serum and the tumor tissues 28 . Furthermore, the normal cells located around the tumor cells might have absorbed some tumor-derived miRNAs ${ }^{29}$, and as a result, only a small amount of the tumor-derived miRNAs that existed in the stromal tissue are thus considered to flow into the blood. Some of miRNAs were also secreted from normal cells, including inflammatory cells, in response to tumor cells ${ }^{30-32}$. Thus, identifying the origin of the serum miRNAs is difficult even in the small extracellular vesicles that carry them in the blood, and no markers specific to tumors have yet been identified. A further investigation will be required to confirm the utility of these 15 miRNAs as prognostic markers in the clinical setting.

MiR-1261 was reported to be associated with migration or invasion of prostate cancer cells ${ }^{33}$ and as a diagnostic marker of hepatocellular carcinoma ${ }^{34}$, however, its association with a diagnosis of osteosarcoma in unclear. In the present study, the serum values of miR-1261 in osteosarcoma patients was significantly higher than those in healthy controls, patients with benign bone tumors, and patients with intermediategrade bone tumors, although there were no significant differences from those of patients with other high-grade bone tumors. The presence of 
histological high-grade malignancies, including osteosarcoma, might be reflected by the high serum values of miR-1261 in patients with bone tumors.

Sponging miR-1273c was reported to be associated with suppression of non-small cell lung cancer ${ }^{35,36}$, however, in the present study, low serum values of miR-1273c were associated with malignant bone tumors, including osteosarcoma, Ewing sarcoma and chondrosarcoma (Grade 2-3). MiR-1273c values were higher in the serum of benign or intermediate-grade bone tumors than in healthy controls, whereas the values were lower in the serum of patients with high-grade bone tumors than in healthy controls, although there were no significant differences in the values among these high-grade bone tumors. The presence of histological high-grade malignancies, including osteosarcoma, might be reflected by the low serum values of miR-1273c that were observed in patients with bone tumors.

Several limitations associated with the present study warrant mention. First, this was a retrospective, small-scale, single-institution study. Second, the peripheral blood findings were not compared between the preoperative time and postoperative time. Changes in the values of miRNA after surgery might indicate tumor-derived miRNAs ${ }^{21-24}$, but, in the present study, the postoperative blood findings for miRNAs were not investigated because the timing of surgery, methods of surgical intervention, and other treatments including chemotherapy, differed among patients. Third, the characteristics concerning age and sex in healthy patients and the patients with other types of bone tumors were not compared with those in osteosarcoma patients since there was only a small number of patients, and also because the predominance of age and sex originally depends on the histological type of bone tumors ${ }^{12}$.

In conclusion, the 15 serum miRNAs evaluated here, including miR-146a-5p, may not be useful markers for the prognosis, and a larger investigation will be required to confirm their actual utility. However, serum miR-1261 may be useful for differentiating histological high-grade bone tumors, including osteosarcoma, from benign or intermediate-grade bone tumors.

\section{Methods}

\section{Patient enrollment}

A total of 30 osteosarcoma patients without metastasis at presentation were retrospectively reviewed from 2007 to 2015 . Patients with osteochondroma $(n=15)$, enchondroma $(n=11)$, and fibrous dysplasia $(n=10)$ as benign tumors; giant cell tumor of bone $(n=28)$, chondromatosis $(n=5)$, and osteoblastoma $(n=3)$ as intermediate-grade tumors, and Ewing sarcoma $(n=26)$, chordoma $(n=8)$, and chondrosarcoma (grade2-3) $(n=6)$ as high-grade tumors, were also included, according to the histological grade in the 2020 WHO classification. In addition, 275 individuals who had no history of cancers were included as healthy controls. Patients with other types of bone tumors and healthy controls were investigated for the comparison of serum miRNAs values at presentation.

All patients underwent standard treatment during follow-up period at National Cancer Center Hospital at Tokyo in Japan. This study was conducted in accordance with the 1975 Declaration of Helsinki. It was approved by the NCCH Institutional Review Board (2004-050, 2013 - 111, 2015 - 266) and the Research Ethics Committee of Medical Corporation Shintokai Yokohama Minoru Clinic (6019-18-3772). Written informed consent was obtained from each participant.

\section{Data collection on patient characteristics}

The age, sex, tumor location (limb or trunk), staging (AJCC 8th edition ${ }^{37}$ ), surgery, chemotherapy, radiation therapy, distant metastasis, local recurrence, follow-up periods, and serum miRNA values at the first visit were retrospectively investigated in osteosarcoma patients using medical records. The age, sex, and serum miRNA values at the first visit in patients with other types of bone tumors and healthy controls were also reviewed using medical records.

\section{A comparison of the survival according to miRNA values}

The serum miRNAs values at the first hospital visit, the follow-up duration, and the oncological outcomes at the final follow-up time, were all investigated. Using receiver operating characteristic (ROC) curves, the optimal threshold of the values of miRNAs were defined, predicting death from disease, distant metastasis, or no evidence of disease. The overall survival, distant metastasis-free survival, and disease-free survival were compared according to the values of miRNAs. The overall survival was defined as the period until the death of the osteosarcoma patient since the diagnosis was made. The distant metastasis-free survival was defined as the period until a distant metastasis was detected by imaging examination. The disease-free survival was defined as the period of no evidence of disease.

\section{A comparison of serum miRNAs values in patients with bone tumors}

For differentiation of osteosarcoma, serum miRNA values at presentation were compared with those in the patients with other types of bone tumors (benign, intermediate-grade, and high-grade) and healthy controls.

\section{Statistical analyses}


Using ROC curves, the optimal threshold of the values of miR-146a-5p, miR-1260a, miR-487b-3p, miR-6720-3p, miR-1260b, miR-4758-3p, miR-46903p, miR-4286, miR-6765-3p, miR-1261, miR-7975, miR-4664-5p, miR-3907, miR-7977, and miR-1273c in osteosarcoma patients were obtained when the Youden index was maximal, which predicted death from disease, distant metastasis, or freedom from disease. Thirty osteosarcoma patients were divided into two groups based on the cut-off values (either a low or high value). The overall survival, distant metastasis-free survival, and disease-free survival was plotted using the Kaplan-Meier method in each group and compared by the log-rank test. P-values of $<0.05$ was considered to indicate statistical significance.

Among the values of each miRNA (miR-146a-5p, miR-1260a, miR-487b-3p, miR-6720-3p, miR-1260b, miR-4758-3p, miR-4690-3p, miR-4286, miR6765-3p, miR-1261, miR-7975, miR-4664-5p, miR-3907, miR-7977, and miR-1273c) in ten bone tumor groups, Kruskal-Wallis test was performed for differentiating of osteosarcoma, and as a post-hoc test, all two groups were compared by Steel-Dwass multiple tests. P-values of < 0.05 was considered to indicate statistical significance. All statistical analyses were performed using EZR (Saitama Medical Center, Jichi Medical University, Saitama, Japan), which is a graphical user interface for the R software program (The R Foundation for Statistical Computing, Vienna, Austria) ${ }^{38}$.

\section{Abbreviations}

Micro-ribonucleic acid: miRNA; AJCC: American Joint Committee on Cancer; Receiver operating characteristic: ROC; Areas under the curve: AUC

\section{Declarations}

\section{Acknowledgements}

We thank Akihiko Yoshida for performing histological examination of all the specimen. In addition, we thank the past and the present other members of our department and associated institution for their work.

\section{Authors' contributions}

HT, NY, and YAr conceived and designed the study. YAr carried out data acquisition. NA provided assistance for data acquisition. NA and AK managed the patients for the appropriate treatment and observed them at the follow-up outpatient clinic. YAr analyzed all the patient's data and wrote the manuscript. HT, NY and YAr contributed to the analysis of the data and critical appraisal. KH, AT, SM, KI, TH, KA, HY, SM, YAs, JM, TO, TY, and $\mathrm{RH}$ supervised the analysis. All authors read and approved the final manuscript.

\section{Competing interests}

The authors declare no conflicts of interest in association with the present study. This research received no specific grant from any funding agency in the public, commercial, or not-for-profit sectors.

\section{Data availability}

All data generated or analyzed during the present study are included in this published article.

\section{Ethics declarations}

This study was conducted in accordance with the 1975 Declaration of Helsinki.

\section{Approval for human experiments}

All patients underwent standard treatment during follow-up period at National Cancer Center Hospital (NCCH) at Tokyo in Japan. This study was conducted in accordance with the 1975 Declaration of Helsinki. It was approved by the NCCH Institutional Review Board (2004-050, 2013-111, 2015-266) and the Research Ethics Committee of Medical Corporation Shintokai Yokohama Minoru Clinic (6019-18-3772). Written informed consent was obtained from each participant.

\section{Consent to participate/Consent to publish}

The consent for partcicipation and publication of the manuscript and the related images from the patients and/or their parents (in case of patients aged $<18$ years old) was obtained by National Cancer Center Hospital at Tokyo in Japan.

\section{References}

1. Saliminejad, K., Khorram Khorshid, H.R., Soleymani Fard, S. \& Ghaffari, S.H. An overview of microRNAs: Biology, functions, therapeutics, and analysis methods. J Cell Physiol. 234(5), 5451-65 (2019). doi: 10.1002/jcp.27486. PMID: 30471116. 
2. Rupaimoole, R., Calin, G.A., Lopez-Berestein, G. \& Sood, A.K. miRNA Deregulation in Cancer Cells and the Tumor Microenvironment. Cancer Discov. 6(3), 235 - 46 (2016). doi: 10.1158/2159-8290.CD-15-0893. PMID: 26865249.

3. Sun, Z. et al. Effect of exosomal miRNA on cancer biology and clinical applications. Mol Cancer. 17(1), 147 (2018). doi: 10.1186/s12943-0180897-7. PMID: 30309355.

4. Pontecorvi, G., Bellenghi, M., Puglisi, R., Carè, A. \& Mattia, G. Tumor-derived extracellular vesicles and microRNAs: Functional roles, diagnostic, prognostic and therapeutic options. Cytokine Growth Factor Rev. 51, 75-83 (2020). doi: 10.1016/j.cytogfr.2019.12.010. PMID: 31924512.

5. Tang, Z., Li, D., Hou, S. \& Zhu, X. The cancer exosomes: Clinical implications, applications and challenges. Int J Cancer. 146(11), 2946-59 (2020). doi: 10.1002/ijc.32762. PMID: 31671207.

6. Chen, Q., Li, Y., Liu, Y., Xu, W. \& Zhu, X. Exosomal Non-coding RNAs-Mediated Crosstalk in the Tumor Microenvironment. Front Cell Dev Biol. 9 , 646864 (2021). doi: 10.3389/fcell.2021.646864. PMID: 33912560.

7. McGuire, A., Brown, J.A. \& Kerin, M.J. Metastatic breast cancer: the potential of miRNA for diagnosis and treatment monitoring. Cancer Metastasis Rev. 34(1), 145 - 55 (2015). doi: 10.1007/s10555-015-9551-7. PMID: 25721950.

8. Ishikawa, D. et al. Expression level of microRNA-449a predicts the prognosis of patients with gastric cancer. Anticancer Res. 40(1), 239 - 44 (2020). doi: 10.21873/anticanres.13945. PMID: 31892572.

9. Amankwah, E.K., Devidas, M., Teachey, D.T., Rabin, K.R. \& Brown, P.A. Six candidate miRNAs associated with early relapse in pediatric B-cell acute lymphoblastic leukemia. Anticancer Res. 40(6), 3147-53 (2020). doi: 10.21873/anticanres.14296. PMID: 32487609.

10. Yu, H., Guan, Z., Cuk, K., Brenner, H. \& Zhang, Y. Circulating microRNA biomarkers for lung cancer detection in Western populations. Cancer Med. 7(10), 4849-62 (2018). doi: 10.1002/cam4.1782. PMID: 30259714.

11. Zeng, Z. et al. Cancer-derived exosomal miR-25-3p promotes pre-metastatic niche formation by inducing vascular permeability and angiogenesis. Nat Commun. 9(1), 5395 (2018). doi: 10.1038/s41467-018-07810-w. PMID: 30568162.

12. Dialani, L., Valerie, A. \& Ian, A.C. WHO Classification of Tumours. Soft Tissue and Bone Tumours. 5th ed. Osteosarcoma. 403-409 (IARC, 2020).

13. Reed, D.R. et al. Treatment pathway of bone sarcoma in children, adolescents, and young adults. Cancer. 123(12), 2206-18 (2017). doi: 10.1002/cncr.30589. Epub 2017 Mar 21. PMID: 28323337.

14. Tsukamoto, S., Errani, C., Angelini, A., \& Mavrogenis, A.F. Current Treatment Considerations for Osteosarcoma Metastatic at Presentation. Orthopedics. 43(5), e345-e358 (2020). doi: 10.3928/01477447-20200721-05. PMID: 32745218.

15. Araki, Y. et al. Delayed initiation of treatment is associated with metastasis of malignant bone tumor. Anticancer Res. 41(6), 2993-99 (2021). doi: 10.21873/anticanres.15081. PMID: 34083290.

16. Hao, H., Chen, L., Huang, D., Ge, J., Qiu, Y. \& Hao, L. Meta-analysis of alkaline phosphatase and prognosis for osteosarcoma. Eur J Cancer Care (Engl). 26(5) (2017). doi: 10.1111/ecc.12536. PMID: 27349943.

17. Araki, Y. et al. Pretreatment Neutrophil Count and Platelet-lymphocyte Ratio as Predictors of Metastasis in Patients With Osteosarcoma. Anticancer Res. 42(2), 1081-89 (2022). doi: 10.21873/anticanres.15570. PMID: 35093910.

18. Ding, W.Z., Liu, K., Li, Z. \& Chen, S.R. A meta-analysis of prognostic factors of osteosarcoma. Eur Rev Med Pharmacol Sci. 24(8), 4103-12 (2020). doi: 10.26355/eurrev_202004_20989. PMID: 32373946

19. Araki, Y. et al. The number of osteoclasts in a biopsy specimen can predict the efficacy of neoadjuvant chemotherapy for primary osteosarcoma. Sci Rep. 11(1), 1989 (2021). doi: 10.1038/s41598-020-80504-w. PMID: 33479294.

20. Wang, J. et al. The Role of miRNA in the diagnosis, prognosis, and treatment of osteosarcoma. Cancer Biother Radiopharm. 34(10), 605 - 13 (2019). doi: 10.1089/cbr.2019.2939. PMID: 31674804.

21. Raimondi, L. et al. Osteosarcoma cell-derived exosomes affect tumor microenvironment by specific packaging of microRNAs. Carcinogenesis. 41(5), 666 - 77 (2020). doi: 10.1093/carcin/bgz130. PMID: 31294446.

22. Huang, C., Sun, Y., Ma, S., Vadamootoo, A.S., Wang, L. \& Jin, C. Identification of circulating miR-663a as a potential biomarker for diagnosing osteosarcoma. Pathol Res Pract. 215(6), 152411 (2019). doi: 10.1016/j.prp.2019.04.003. PMID: 30987831.

23. Fujiwara, T. et al. Clinical significance of circulating miR-25-3p as a novel diagnostic and prognostic biomarker in osteosarcoma. Oncotarget. 8(20), 33375-92 (2017). doi: 10.18632/oncotarget.16498. PMID: 28380419.

24. Huang, C., Wang, Q., Ma, S., Sun, Y., Vadamootoo, A.S. \& Jin, C. A four serum-miRNA panel serves as a potential diagnostic biomarker of osteosarcoma. Int J Clin Oncol. 24(8), 976 - 82 (2019). doi: 10.1007/s10147-019-01433-x. PMID: 31111286.

25. Asano, N. et al. A serum microRNA classifier for the diagnosis of sarcomas of various histological subtypes. Nat Commun. 10(1), 1299 (2019). doi: 10.1038/s41467-019-09143-8. PMID: 30898996.

26. Araki, Y. et al. Osteosarcoma-Derived Small Extracellular Vesicles Enhance Tumor Metastasis and Suppress Osteoclastogenesis by miR-146a5p. Front Oncol. 11, 667109 (2021). doi: 10.3389/fonc.2021.667109. PMID: 34017686.

27. https://www.nedo.go.jp/news/press/AA5_100275.html

Page $7 / 12$ 
28. Boele, J. et al. PAPD5-mediated 3' adenylation and subsequent degradation of miR-21 is disrupted in proliferative disease. Proc Natl Acad Sci U S A. 111(31), 11467-72 (2014). doi: 10.1073/pnas.1317751111. PMID: 25049417.

29. Li, K. et al. Advances, challenges, and opportunities in extracellular RNA biology: insights from the NIH exRNA Strategic Workshop. JCI Insight. 3(7), e98942 (2018). doi: 10.1172/jci.insight.98942. PMID: 29618663.

30. Engin, A. Dark-Side of Exosomes. Adv Exp Med Biol. 1275, 101 - 31 (2021). doi: 10.1007/978-3-030-49844-3_4. PMID: 33539013.

31. Robbins, P.D. \& Morelli, A.E. Regulation of immune responses by extracellular vesicles. Nat Rev Immunol. 14(3), 195-208 (2014). doi: 10.1038/nri3622. PMID: 24566916.

32. Sinkovics, J.G. Molecular biology of oncogenic inflammatory processes. I. Non-oncogenic and oncogenic pathogens, intrinsic inflammatory reactions without pathogens, and microRNA/DNA interactions (Review). Int J Oncol. 40(2), 305 - 49 (2012). doi: 10.3892/ijo.2011.1248. PMID: 22076306.

33. He, J.H., et al. Snail-activated long non-coding RNA PCA3 up-regulates PRKD3 expression by miR-1261 sponging, thereby promotes invasion and migration of prostate cancer cells. Tumour Biol. 37, 16163-76 (2016). doi: 10.1007/s13277-016-5450-y. PMID: 27743381.

34. Lyu, N. et al. Ferroptosis is involved in the progression of hepatocellular carcinoma through the circ0097009/miR-1261/SLC7A11 axis. Ann Transl Med. 9(8), 675 (2021). doi: 10.21037/atm-21-997. PMID: 33987373.

35. Yu, H. et al. RNA sequencing uncovers the key long non-coding RNAs and potential molecular mechanism contributing to XAV939-mediated inhibition of non-small cell lung cancer. Oncol Lett. 17(6), 4994-5004 (2019). doi: 10.3892/ol.2019.10191. PMID: 31186710.

36. Ma, W., Kang, Y., Ning, L., Tan, J., Wang, H. \& Ying, Y. Identification of microRNAs involved in gefitinib resistance of non-small-cell lung cancer through the insulin-like growth factor receptor 1 signaling pathway. Exp Ther Med. 14(4), 2853-62 (2017). doi: 10.3892/etm.2017.4847. PMID: 28912847.

37. Amin, MB. et al. AJCC Cancer Staging Manual. 8th ed. Bone. 471-486 (Springer, 2017).

38. Kanda, Y. Investigation of the freely available easy-to-use software 'EZR' for medical statistics. Bone Marrow Transplant. 48(3), 452-8 (2013).

\section{Figures}




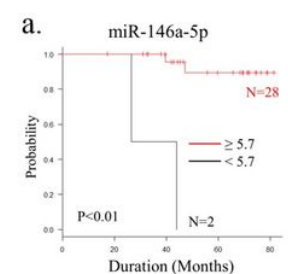

b.
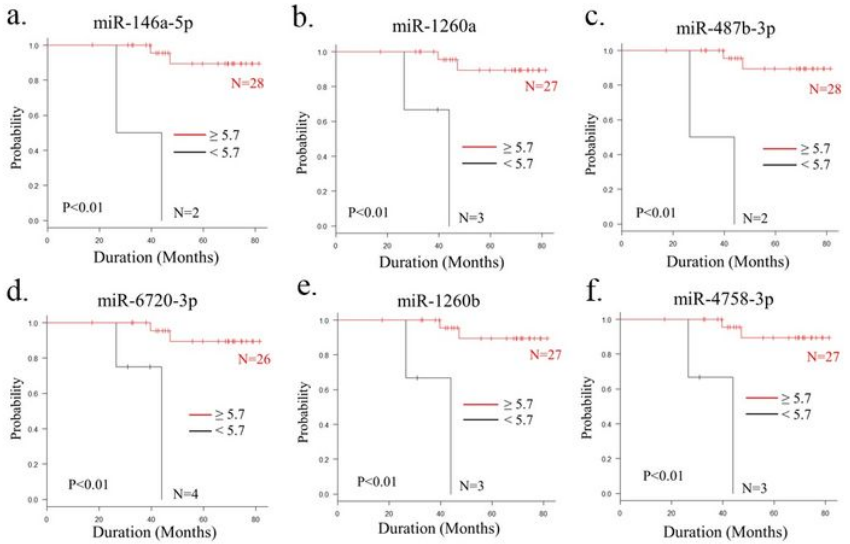

e.

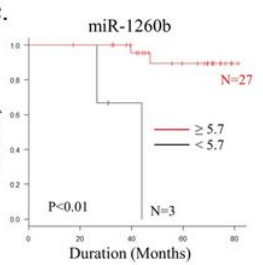

f.
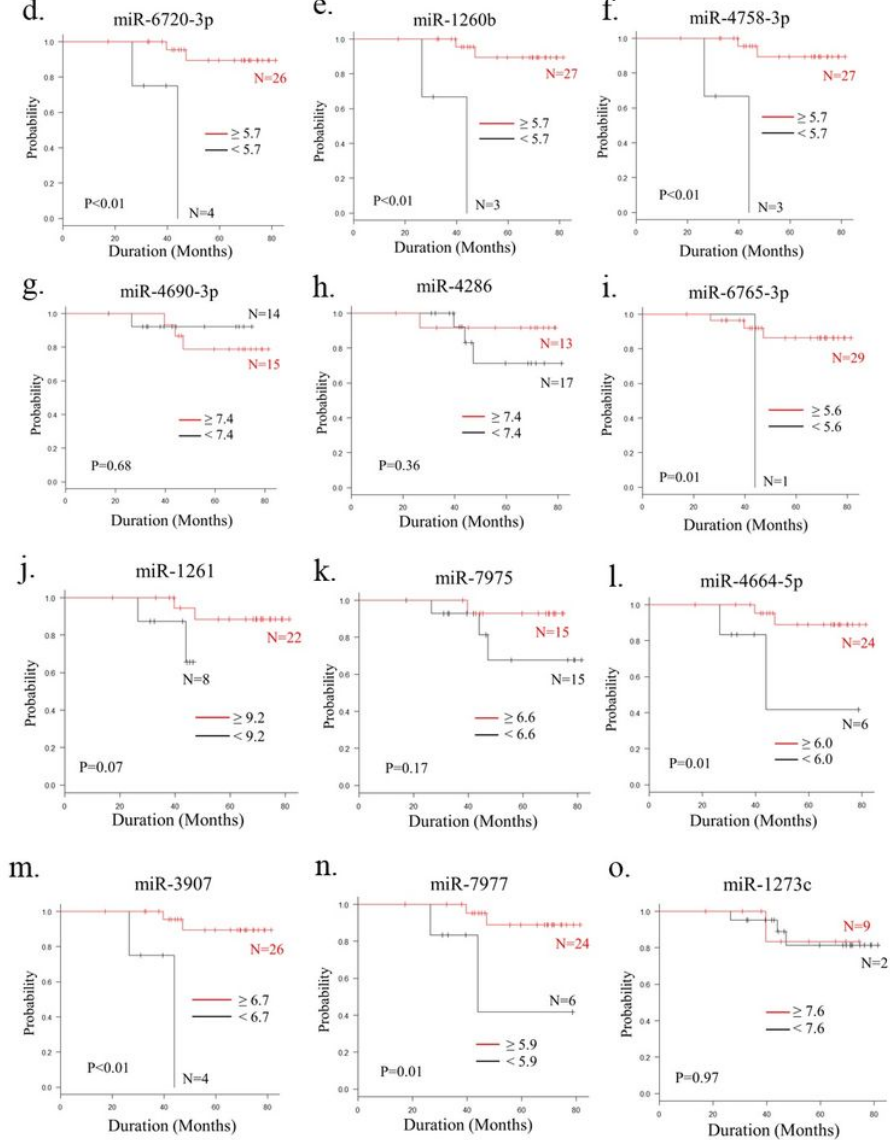

n.
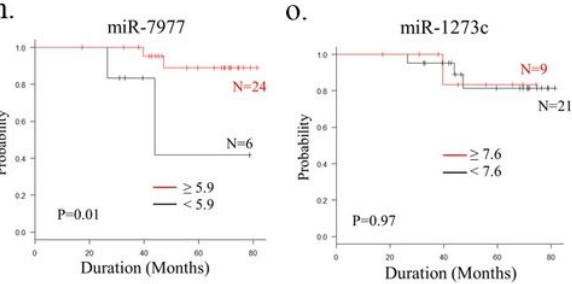

\section{Figure 1}

A comparison of the overall survival according to miRNA values. a.miR-146a-5p, b.miR-1260a, c.miR-487b-3p, d.miR-6720-3p, e.miR-1260b, f.miR4758-3p, g.miR-4690-3p, h.miR-4286, i.miR-6765-3p, j.miR-1261, k.miR-7975, I.miR-4664-5p, m.miR-3907, n.miR-7977, o.miR-1273c. 

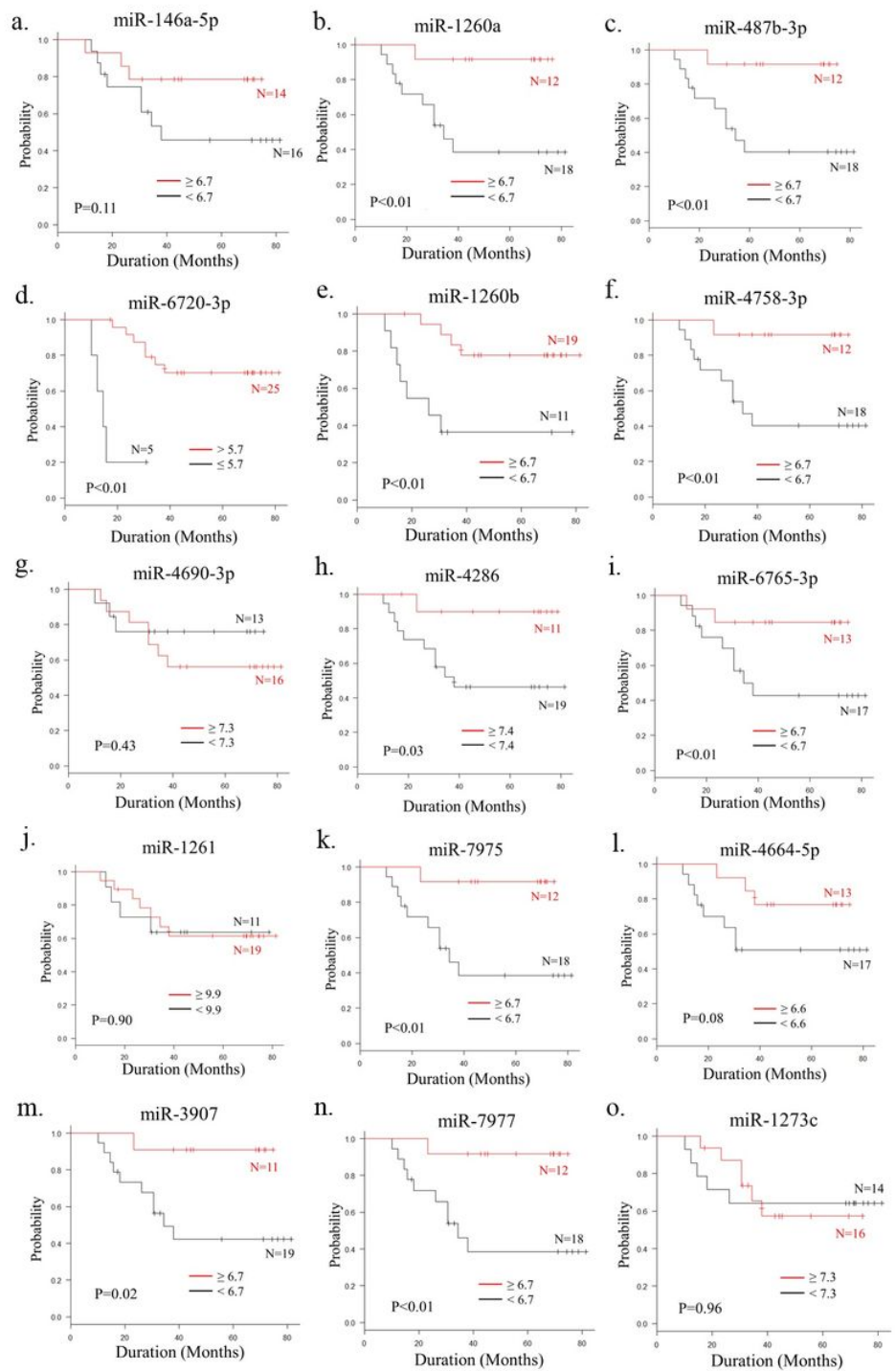

Figure 2

A comparison of the distant metastasis-free survival according to miRNA values. a.miR-146a-5p, b.miR-1260a, c.miR-487b-3p, d.miR-6720-3p, e.miR-1260b, f.miR-4758-3p, g.miR-4690-3p, h.miR-4286, i.miR-6765-3p, j.miR-1261, k.miR-7975, I.miR-4664-5p, m.miR-3907, n.miR-7977, o.miR1273c. 

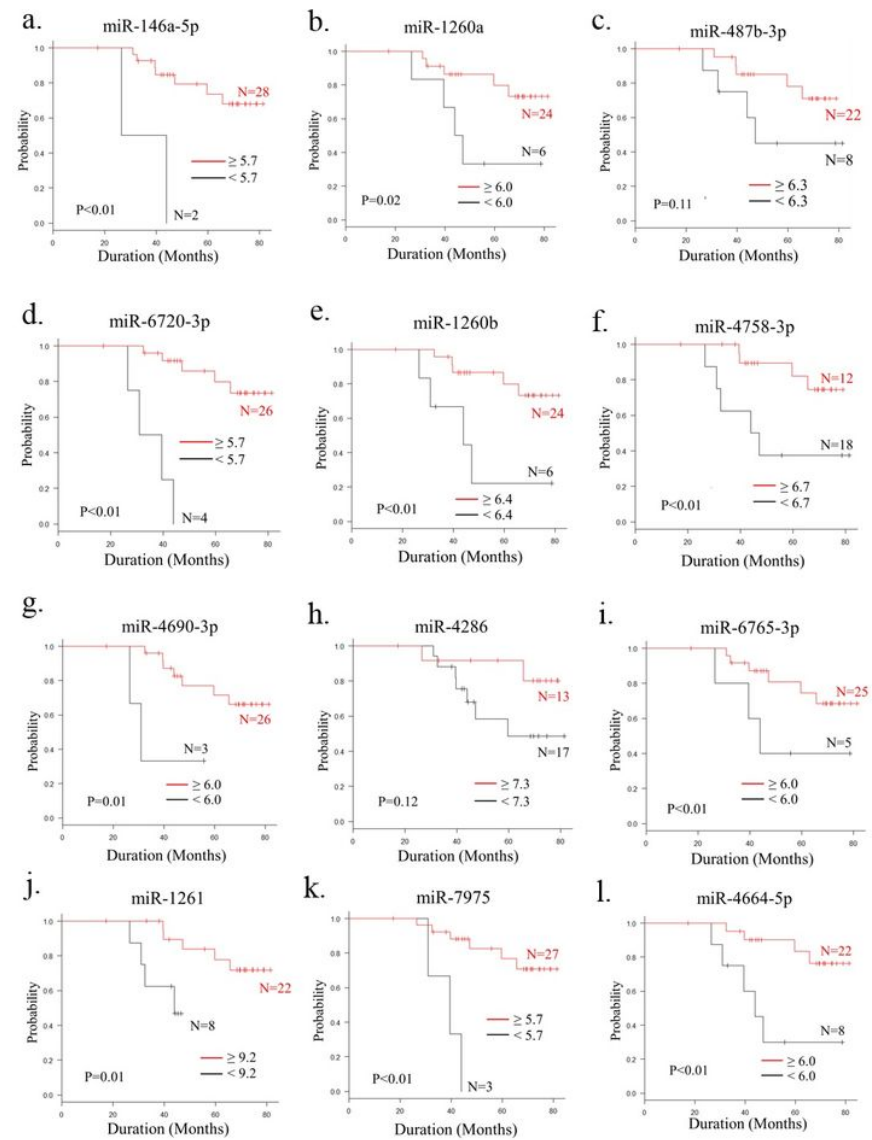

$\mathrm{k}$.
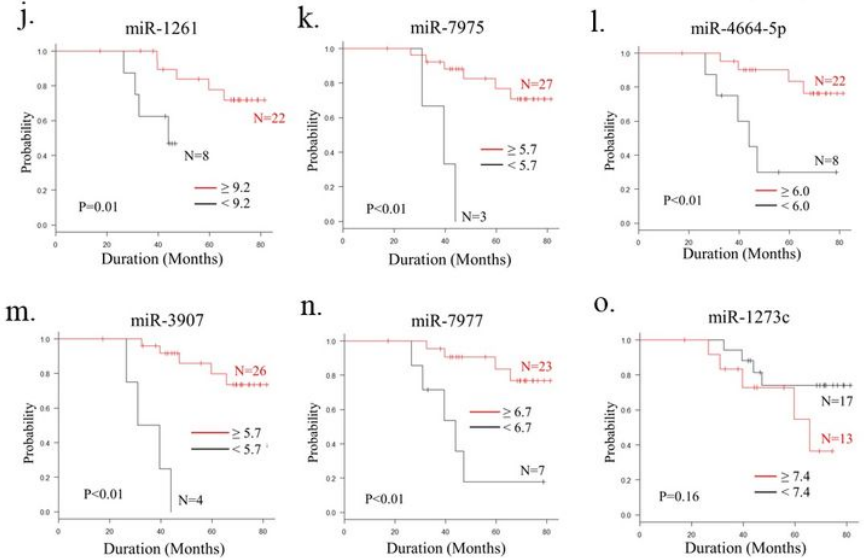

\section{Figure 3}

A comparison of the disease-free survival according to miRNA values. a.miR-146a-5p, b.miR-1260a, c.miR-487b-3p, d.miR-6720-3p, e.miR-1260b, f.miR-4758-3p, g.miR-4690-3p, h.miR-4286, i.miR-6765-3p, j.miR-1261, k.miR-7975, I.miR-4664-5p, m.miR-3907, n.miR-7977, o.miR-1273c. 

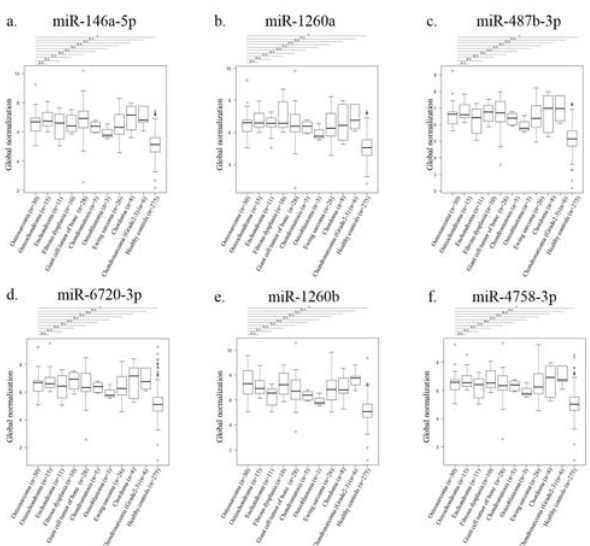

f. $\quad$ miR-4758-3p
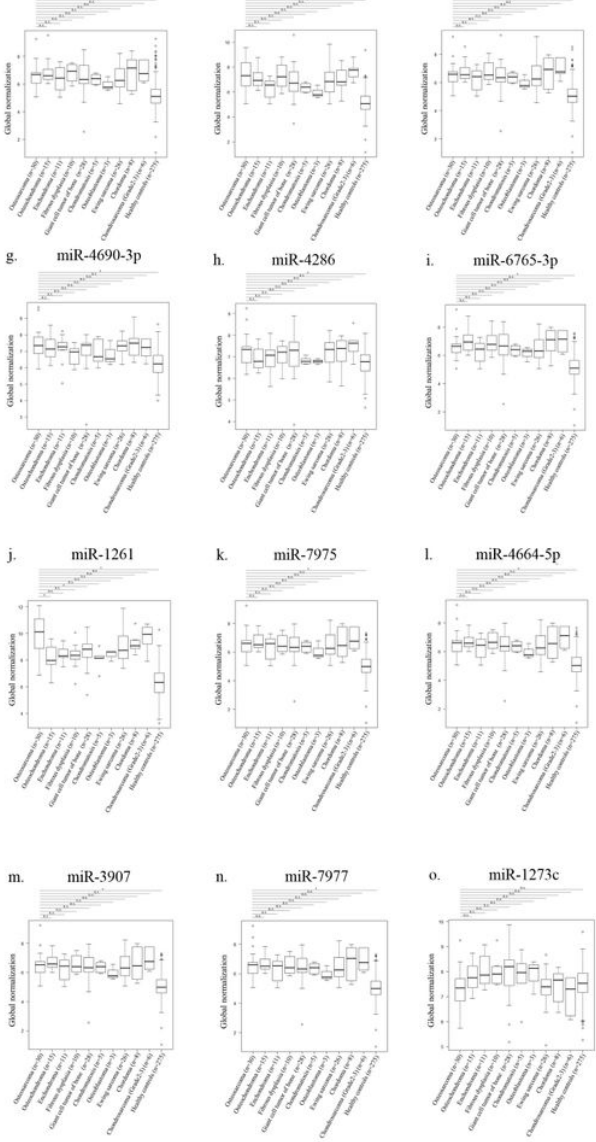

\section{Figure 4}

A comparison of serum miRNAs values in patients with bone tumors and healthy controls. a.miR-146a-5p, b.miR-1260a, c.miR-487b-3p, d.miR6720-3p, e.miR-1260b, f.miR-4758-3p, g.miR-4690-3p, h.miR-4286, i.miR-6765-3p, j.miR-1261, k.miR-7975, I.miR-4664-5p, m.miR-3907, n.miR-7977, o.miR-1273c. An asterisk ( ${ }^{*}$ ) means p-values of $<0.05$, and n.s. means no significant difference. 